PROCEEDINGS OF THE

AMERICAN MATHEMATICAL SOCIETY

Volume 129, Number 7, Pages 2187-2189

S 0002-9939(01)06098-1

Article electronically published on February 15, 2001

\title{
A SHORT PROOF THAT HYPERSPACES OF PEANO CONTINUA ARE ABSOLUTE RETRACTS
}

\author{
SERGEY ANTONYAN
}

(Communicated by Alan Dow)

ABstract. We give a short proof of Wojdyslawski's famous theorem.

Theorem (Wojdyslawski [6]). Let $X$ be a Peano continuum. Then the hyperspace $2^{X}$ of all nonempty compact subsets of $X$ is an absolute retract for metric spaces.

This result is an essential step in the proof of the Curtis-Schori-West Hyperspace Theorem to the effect that $2^{X}$ is a Hilbert cube for any Peano continuum $X$ (see, e.g., the book of van Mill [5, §8.4]). Wojdyslawski's original proof is rather complicated [6]. A simpler proof was suggested later on by Kelley [4, which is, however, based on a difficult Lefschetz-Dugundji characterization of metric ANR's (see [5. Theorem 5.2.1]). Yet another proof, also based on the Lefschetz-Dugundji characterization, can be found in 5, $\$ 5.3]$. Our proof is elementary and it does not rely on the Lefschetz-Dugundji criterion.

Proof. Let $d$ be any compatible metric on $X$ and let $d_{H}$ be the Hausdorff metric on $2^{X}$. Assume that $(Y, \rho)$ is a metric space, $A$ is a closed subset of $Y$ and $f: A \rightarrow 2^{X}$ is a continuous map. Following [3], choose a canonical cover $\omega$ of $Y \backslash A$ in $Y$, that is to say: (1) $\omega$ is an open cover of $Y \backslash A$, locally finite in $Y \backslash A$; (2) for each neighborhood $V$ of a point $a \in A$ in $Y$ there exists a neighborhood $S$ of $a$ in $Y$ contained in $V$, such that every element $U \in \omega$ which meets $S$ is contained in $V$. We note that the second condition implies that every neighborhood of any boundary point of $A$ in $Y$ contains infinitely many open sets in $\omega$ (see [2] Ch. III, §1]).

Let $\mathcal{N}(\omega)$ denote the nerve of $\omega$ endowed with the $C W$ topology. We will denote by $p_{U}$ the vertex of $\mathcal{N}(\omega)$ corresponding to $U \in \omega$. Then according to [3], there exist a Hausdorff space $Z$ and a continuous map $\mu: Y \rightarrow Z$ with the following properties:

(a) $Z$ as a set coincides with the disjoint union $A \cup \mathcal{N}(\omega)$;

(b) $A$ is closed in $Z$ and the restriction $\left.\mu\right|_{A}$ is the identical homeomorphism;

(c) $Z \backslash A=\mathcal{N}(\omega)$ is taken with its $C W$ topology and $\mu(Y \backslash A) \subset Z \backslash \mu(A)$;

(d) a base of neighborhoods of $a \in A$ in $Z$ is determined by selecting a neighborhood $W$ of $a$ in $Y$ and taking in $Z$ the set $W \cap A$ together with the closed

Received by the editors October 29, 1999 and, in revised form, September 5, 2000.

2000 Mathematics Subject Classification. Primary 54B20; Secondary 54C55.

Key words and phrases. Peano continuum, absolute retract, hyperspace, canonical cover, nerve.

The author was partially supported by PAPIIT (UNAM) research grant IN105800. 
star of every vertex $p_{U}$ of $\mathcal{N}(\omega)$ corresponding to a set $U \in \omega$ with $U \subset W$. This neighborhood is denoted by $\widetilde{W}$.

It is sufficient to prove that $f$ extends to a continuous map $F: Z \rightarrow 2^{X}$; then the map $\Phi=F \mu: Y \rightarrow 2^{X}$ will be the desired extension of $f$.

Let $\mathcal{N}_{k}(\omega)$ denote the $k$-skeleton of $\mathcal{N}(\omega)$. First we extend $f$ to a map $f_{0}$ : $A \cup \mathcal{N}_{0}(\omega) \rightarrow 2^{X}$ as follows: in every set $U \in \omega$ we select a point $x_{U}$ and then choose a point $a_{U} \in A$ such that $\rho\left(x_{U}, a_{U}\right)<2 \rho\left(x_{U}, A\right)$. Set $f_{0}\left(p_{U}\right)=f\left(a_{U}\right)$ and $f_{0}(a)=f(a)$ for $a \in A$. It is readily seen that $f_{0}$ is continuous. Now we will extend $f_{0}$ over each simplex of $\mathcal{N}(\omega)$ and thus we obtain the desired map $F$. Since $2^{X}$ is a Peano continuum [5, Proposition 5.3.10], it is path-connected and locally pathconnected by a well-known result of Mazurkiewicz (see [5, Theorem 5.3.13]). For any two points $B, C \in 2^{X}$ we select a path $l_{B, C}:[0,1] \rightarrow 2^{X}$ such that $l_{B, C}(0)=B$, $l_{B, C}(1)=C$ and

$$
\operatorname{diam} l_{B, C}([0,1])<2 \inf \{\operatorname{diam} \gamma([0,1]): \gamma \text { is a path from } B \text { to } C\} .
$$

We now extend $f_{0}$ to a map $f_{1}: A \cup \mathcal{N}_{1}(\omega) \rightarrow 2^{X}$ by the rule: $f_{1}(a)=f_{0}(a)$ for $a \in A$ and $f_{1}\left(t p_{U}+(1-t) p_{V}\right)=l_{f_{0}\left(p_{U}\right), f_{0}\left(p_{V}\right)}(t), \quad 0 \leq t \leq 1$. One needs to prove $f_{1}$ continuous only at points of $A$. Let $a \in A, \varepsilon>0$ and $O(f(a), \delta)$ be the $\delta$-neighborhood of $f(a)$ in $2^{X}$. By the local path-connectedness of $2^{X}$, there is a path-connected neighborhood $Q$ of $f_{0}(a)=f(a)$ contained in $O(f(a), \varepsilon / 8)$. By continuity of $f_{0}$, there exists a neighborhood of $a$ in $Z$ of the form $\widetilde{W}$ such that $f_{0}\left(\widetilde{W} \cap\left(A \cup \mathcal{N}_{0}(\omega)\right)\right) \subset Q$. Then $f_{1}\left(\widetilde{W} \cap\left(A \cup \mathcal{N}_{1}(\omega)\right)\right) \subset O(f(a), \varepsilon)$. Indeed, if $z=t p_{U}+(1-t) p_{V} \in \widetilde{W} \cap \mathcal{N}_{1}(\omega)$, then $f_{0}\left(p_{U}\right), f_{0}\left(p_{V}\right) \in Q$; so $Q$ contains a path $\gamma$, connecting $f_{0}\left(p_{U}\right)$ and $f_{0}\left(p_{V}\right)$. Hence diam $\gamma([0,1])<\varepsilon / 4$, which implies that diam $l_{f_{0}\left(p_{U}\right), f_{0}\left(p_{V}\right)}([0,1])<\varepsilon / 2$. Then $d_{H}\left(f_{1}(z), f_{1}(a)\right)<\varepsilon$ because $f_{1}(z) \in$ $l_{f_{0}\left(p_{U}\right), f_{0}\left(p_{V}\right)}([0,1])$.

Now suppose that a continuous extension $f_{k}: A \cup \mathcal{N}_{k}(\omega) \rightarrow 2^{X}$ of $f_{k-1}, k \geq 1$ has already been constructed. We shall construct an extension $f_{k+1}: A \cup \mathcal{N}_{k+1}(\omega) \rightarrow 2^{X}$ of $f_{k}$. Let $\sigma$ be any $(k+1)$-dimensional simplex in $\mathcal{N}(\omega)$. Let $\mathbb{B}^{k+1}$ be the $(k+1)$ dimensional Euclidean closed unit ball and $\mathbb{S}^{k}$ be its boundary sphere. We aim at applying the following well-known easy fact: for every $k \geq 1$ there exists a continuous function $r: \mathbb{B}^{k+1} \rightarrow 2^{\mathbb{S}^{k}}$ such that $r(y)=\{y\}$ for all $y \in \mathbb{S}^{k}$ (see, e.g., [5, Proposition 5.3.11]). To this end, it is convenient to identify the pair $(\sigma, \partial \sigma)$ with $\left(\mathbb{B}^{k+1}, \mathbb{S}^{k}\right)$. Then the preceding fact insures the existence of a continuous map $r_{\sigma}: \sigma \rightarrow 2^{\partial \sigma}$ such that $r_{\sigma}(z)=\{z\}$ for every $z \in \partial \sigma$. The map $g_{\sigma}: 2^{\partial \sigma} \rightarrow 2^{X}$ defined by $g_{\sigma}(C)=\bigcup_{c \in C} f_{k}(c)$ is continuous [5] Corollary 5.3.7]. Then $f_{\sigma}=g_{\sigma} r_{\sigma}$ : $\sigma \rightarrow 2^{X}$ is a continuous extension of $\left.f_{k}\right|_{\partial \sigma}$. Now we set $f_{k+1}(z)=f_{\sigma}(z)$ if $z \in \sigma$, and $f_{k+1}(a)=f_{k}(a)$ if $a \in A$. Then $f_{k+1}$ extends $f_{k}$ and is continuous on $\mathcal{N}_{k+1}(\omega)$. We define the map $F: Z \rightarrow 2^{X}$ as follows: $F(z)=f_{k}(z)$ whenever $z \in A \cup \mathcal{N}_{k}(\omega)$. Clearly, $F$ is continuous on $\mathcal{N}(\omega)$. Let us check its continuity at points of $A$. Let $a \in A$ and $\varepsilon>0$. By continuity of $f_{1}$, there is a neighborhood of $a$ in $Z$ of the form $\widetilde{W}$ such that $f_{1}\left(\widetilde{W} \cap\left(A \cup \mathcal{N}_{1}(\omega)\right)\right) \subset O(f(a), \varepsilon)$. We claim that $F(\widetilde{W}) \subset O(f(a), \varepsilon)$. We shall prove by induction on the dimension of $\sigma$ that $F(\sigma) \subset O(f(a), \varepsilon)$ for every simplex $\sigma \subset \widetilde{W}$. If $\operatorname{dim} \sigma=1$, then $F(\sigma)=f_{1}(\sigma) \subset O(f(a), \varepsilon)$. Assume that the claim is true for all simplices $s \subset \widetilde{W}$ with $\operatorname{dim} s \leq k$. Let $\sigma \subset \widetilde{W}, \operatorname{dim} \sigma=k+1$ and $z \in \sigma$. As $F(z)=f_{k+1}(z)=g_{\sigma}\left(r_{\sigma}(z)\right)$, we have $F(z)=\bigcup_{c \in r_{\sigma}(z)} f_{k}(c)$. But $d_{H}\left(f_{k}(c), f(a)\right)<\varepsilon$ for all $c \in \partial \sigma$, and in particular, for all $c \in r_{\sigma}(z)$. This 
yields that $d_{H}\left(\bigcup_{c \in r_{\sigma}(z)} f_{k}(c), f(a)\right)<\varepsilon$, i.e., $d_{H}(F(z), f(a))<\varepsilon$, completing the inductive step.

The reader can easily observe that the same proof serves also for Curtis' theorem [1, Theorem 1.6] on growth hyperspaces $\mathcal{G} \subset 2^{X}$, where $X$ is any connected and locally continuum-connected metrizable space.

\section{REFERENCES}

1. D. W. Curtis, Hyperspaces of noncompact metric spaces, Compositio Math. 40 (1980), 139152. MR 81c:54009

2. K. Borsuk, Theory of retracts, PWN-Polish Scientific Publishers, Warsaw, 1967. MR 35:7306

3. J. Dugundji, An extension of Tietze's theorem, Pac. J. Math. 1 (1951), 353-367. MR 13:373c

4. J. Kelley, Hyperspaces of a continuum, Trans. Amer. Math. Soc. 52 (1942), 22-36. MR 3:315b

5. J. van Mill, Infinite-dimensional topology. Prerequisites and Introduction, North Holland, 1989. MR 90a:57025

6. M. Wojdyslawski, Retracts absolutes et hyperespaces des continus, Fund. Math. 32 (1939), $184-192$.

Departamento de Matemáticas, Facultad de Ciencias, unam, Circuito Exterior, C.U., 04510, MÉxico D.F., MÉxico

E-mail address: antonyan@servidor.unam.mx 